\section{AB011. The regulatory effects of androgen in wound healing of the prostatic urethra after thulium laser resection of the prostate}

\author{
Shujie Xia \\ Department of Urology, Shanghai First People's Hospital, Shanghai \\ 200080, China
}

Background: This research aims to determine the role of androgen in wound healing of the prostatic urethra after thulium laser resection of the prostate (TmLRP). Further, the change of basal cell proliferation and differentiation as well as macrophage polarization and the inflammatory response influenced by androgen were explored. Moreover, the macrophage cytokines effected by finasteride were detected in urine specimens from thulium laser prostatectomy patients, thus to reveal the mechanism of androgen in wound repair process after prostatectomy.

Methods: (I) Twenty-four beagles that received TmLRPs were randomly distributed into a castration group, a testosterone undecanoate (TU) group and a control group. The prostate wound was assessed once a week using a cystoscope. Histological analysis was then carried out to study the re-epithelialization of the prostatic urethra in each group; (II) immunohistochemical and immunofluorescence techniques were used to determine the basal cell proliferation and differentiation influenced by androgen; (III) the macrophage polarization and inflammatory response in the wound tissue and urine was also investigated; (IV) the macrophage cytokines effected by finasteride were detected in urine specimens from thulium laser prostatectomy patients by enzyme-linked immunosorbent assay (ELISA).

Results: (I) The healing of the prostatic urethra after a TmLRP was more rapid in the castration group and slower in the TU group than that in the control group; (II) castration accelerated re-epithelialization by promoting basal cell proliferation in the wound surface and beneath the wound and by accelerating the differentiation of basal cells into urothelial cells; (III) castration reduced the duration of the inflammatory phase and induced the conversion of M1 macrophages to M2 macrophages, thus accelerating the maturation of the wound. By contrast, androgen supplementation enhanced the inflammatory response and prolonged the inflammatory phase. Moreover, the antiinflammatory phase was delayed and weakened. TNF- $\alpha$, IL-6 and IL-12 that secreted by M1 were higher than the other two groups, and IL-10 and TGF-1 that secreted by M2 were obviously postponed compared with the other two groups; (IV) in the urine specimens from thulium laser prostatectomy patients, the expression of TNF- $\alpha$, IL- 6 and IL-12 were lower while the expression of IL-10 and TGF-1 were higher under the influence of finasteride.

Conclusions: (I) Androgen deprivation promotes reepithelialization of the wound, regulates the inflammatory response, and accelerates wound healing of the prostatic urethra after a TmLRP; (II) castration accelerates reepithelialization by promoting basal cell proliferation in the wound surface and beneath the wound and by accelerating the differentiation of basal cells into urothelial cells; (III) androgen inhibits the prostatic urethra wound healing by regulating the macrophage polarization; (IV) this research established foundation to reveal the mechanism and application of anti-androgen in the treatment of wound healing after prostatectomy.

Keywords: Basal cell; benign prostatic hyperplasia; androgen; wound healing; macrophage

doi: 10.21037/tau.2018.AB011

Cite this abstract as: $\mathrm{Xia} \mathrm{S}$. The regulatory effects of androgen in wound healing of the prostatic urethra after thulium laser resection of the prostate. Transl Androl Urol 2018;7(Suppl 5):AB011. doi: 10.21037/tau.2018.AB011 